\title{
ESPECIES DE ENCARSIA FÖRSTER (HYMENOPTERA: APHELINIDAE) PARASITOIDES DE LA MOSQUITA BLANCA ALEUROTHRIXUS CHIVELENSIS (SAMPSON \& DREWS) (HEMIPTERA: ALEYRODIDAE) EN TAMAULIPAS, MÉXICO
}

\author{
SVETlana N. MYARTSEVA, ENRiQue RUIZ-CANCINO* y JuANA M. CORONADO-BLANCO
}

Facultad de Ingeniería y Ciencias, Universidad Autónoma de Tamaulipas, 87149 Ciudad Victoria, Tamaulipas, México.<eruiz@uat.edu.mx>

Recibido: 01/10/2014; aceptado: 03/12/2014

\begin{abstract}
Myartseva, S. N., Ruiz-Cancino, E. \& Coronado-Blanco, J. M. 1015. Especies de Encarsia Förster (Hymenoptera: Aphelinidae) parasitoides de la mosquita blanca Aleurothrixus chivelensis (Sampson \& Drews) (Hemiptera: Aleyrodidae) en Tamaulipas, México. Acta Zoológica Mexicana (n. s.), 31(1): 109-112.
\end{abstract}

RESUMEN. Tres especies de parasitoides fueron obtenidas de la mosquita blanca Aleurothrixus chivelensis (Sampson \& Drews) en el Estado de Tamaulipas, México: Encarsia americana (DeBach \& Rose), E. dimai Myartseva y E. altamira sp. nov., la cual se describe en este artículo y pertenece al complejo de especies meritoria. Aleurothrixus chivelensis es un nuevo registro para Tamaulipas, se registra una planta parásita como hospedera y sus parasitoides.

Palabras clave: Afelínidos, mosquita blanca, planta parasítica.

\section{INTRODUCCIÓN}

Aphelinidae es una familia de Chalcidoidea que cuenta con unas 1,350 especies de 36 géneros (Noyes 2013). Muchas especies de Aphelinidae han sido usadas en proyectos de control biológico clásico contra especies plaga de las familias Diaspididae y Aleyrodidae (Hemiptera) (Greathead 1986, Rosen \& DeBach 1990, Arredondo \& Rodríguez 2008). En México se conocen 190 especies de 13 géneros de Aphelinidae (Myartseva et al. 2012, Myartseva et al. 2013, Kim \& Heraty 2012; Myartseva 2014).

Encarsia Förster es el género más diverso de Aphelinidae, es cosmopolita, cuenta con más de 400 especies e incluye varias de importancia económica como parasitoides de hemípteros plaga. En las últimas dos décadas, esta familia se ha estudiado intensivamente en varios países: en India se conocen 83 especies (28 nuevas en dicho período) (Hayat 1998, Hayat 2012), en China se registran 76 especies (38 nuevas) (Huang \& Polaszek 1998), en Australia se distribuyen 94 especies (72 nuevas) (Schmidt \& Polaszek 2007) mientras que en México se han reportado 95 especies (56 nuevas) (Myartseva \& Evans 2008; Myartseva et al. 2012, Myartseva et al. 2013, Myartseva 2014). El análisis de estas investigaciones sugiere la megadiversidad del género Encarsia a nivel mundial. Después de 80 años de estudio se ha encontrado que ataca menos de 20 especies de mosquitas blancas plaga, todas de la subfamilia Aleyrodinae (Polaszek et al. 2009).
Myartseva, S. N., Ruiz-Cancino, E. \& Coronado-Blanco, J. M. 1015. Species of Encarsia Förster (Hymenoptera: Aphelinidae) parasitoids of the whitefly Aleurothrixus chivelensis (Sampson \& Drews) (Hemiptera: Aleyrodidae) in Tamaulipas, Mexico. Acta Zoológica Mexicana (n. s.), 31(1): 109-112.

ABSTRACT. Three species of parasitoids were reared from the whitefly Aleurothrixus chivelensis in Tamaulipas, Mexico: Encarsia americana (DeBach \& Rose), E. dimai Myartseva and E. altamira sp. nov., which is described in this article, and belongs to the meritoria speciescomplex. Aleurothrixus chivelensis is a new record for Tamaulipas, one new parasitic plant host is recorded, and its parasitoids.

Key words: Aphelinids, whitefly, parasitic plant.

La mayoría de las especies de Encarsia en México (74) se han registrado como parasitoides de mosquitas blancas mientras que en Diaspididae se reportan 16 especies. Varias especies de Encarsia parasitan mosquitas blancas y escamas armadas que se asocian con Citrus spp., Psidium guajava L. y Pithecellobium dulce (Roxb.) Benth., 15, 10 y 7 especies, respectivamente (Myartseva 2007, Myartseva et al. 2012). Diez especies de Encarsia pueden tener importancia económica para el desarrollo del control biológico de plagas de cítricos, de otros frutales y ornamentales (Myartseva et al. 2013).

En el catálogo de Encarsia del mundo, Heraty et al. (2007) enlistaron 30 grupos de especies, incluyendo el complejo meritoria con cinco especies (Polaszek et al. 2004). En estos grupos, las especies comparten una combinación variada de caracteres morfológicos. En México, Encarsia está representado por 20 grupos de especies. En este trabajo se describe la sexta especie del complejo meritoria.

Aleurothrixus Quaintance \& Baker (Aleyrodidae: Aleyrodinae) contiene 20 especies, es un género del Nuevo Mundo, del cual A. floccosus es la única especie cosmopolita. A. chivelensis es la otra especie que se encuentra en México, fue descrita en 1941 de Oaxaca como Hempelia chivelensis, colectada en Persea sp. (Lauraceae). En 2005, Hempelia fue considerado sinónimo junior de Aleurothrixus por lo que $H$. chivelensis fue transferida a dicho género. Esta especie también se encontró en Belice 
(Martin 2005). En este artículo se describe E. altamira sp. nov., obtenida de $A$. chivelensis y se reporta a esta especie de mosquita blanca para el Estado de Tamaulipas.

\section{MATERIALES Y MÉTODOS}

Se colectaron "pupas" de mosquitas blancas de hojas de plantas parásitas de árboles en Altamira, Tamaulipas, en mayo de 2013 y se llevaron al laboratorio en la UAT, donde se colocaron en frascos de plástico. Las avispas parasitoides que emergieron se colocaron en frascos con alcohol al 75\%. Algunos especímenes fueron montados en laminillas en bálsamo de Canadá y se estudiaron con un microscopio compuesto Leica CME. Las medidas de las estructuras morfológicas se efectuaron con una magnificación de 400x. Las figuras de la nueva especie se prepararon con una magnificación de $7 \times 40$, usando el aparato de dibujo PA-4. Se utilizaron diversas claves de especies de Encarsia (Hayat 1998, Hayat 2012; Huang \& Polaszek 1998; Myartseva et al. 2012). El material tipo está depositado en el Museo de Investigación Entomológica de la Universidad de California - Riverside, Estados Unidos (EU) (UCRC), en el Museo de Insectos de la Facultad de Ingeniería y Ciencias de la UAT en Ciudad Victoria, Tamaulipas, México (UAT) y en el Instituto Zoológico de la Academia de Ciencias de Rusia en San Petersburgo (ZISP). Las plantas fueron identificadas por los doctores Jacinto Treviño-Carreón y Arturo Mora-Olivo (UAT) y las mosquitas blancas por los doctores Vicente E. Carapia-Ruiz (Universidad Autónoma del Estado de Morelos) e Ilya A. Gavrilov-Zimin del Instituto Zoológico (ZISP).

\section{RESULTADOS Y DISCUSIÓN}

La especie de mosquita blanca se identificó como Aleurothrixus chivelensis (Sampson \& Drews, 1941) y la hospedera como Struthanthus sp. (Loranthaceae), una planta parásita conocida como matapalo, también se identificó el árbol atacado por esta planta parásita - la guácima Guazuma ulmifolia Lam. (Sterculiaceae). Los parasitoides corresponden a tres especies de Encarsia: E. americana, E. dimai y E. altamira sp. nov. A continuación se anotan los hospederos de las mosquitas blancas, la distribución geográfica de los himenópteros parasitoides y la descripción de la nueva especie.

\section{Encarsia americana (DeBach \& Rose)}

Material. México, Tamaulipas, Altamira, 5 hembras, ex Aleurothrixus chivelensis (Sampson \& Drews) de Struthanthus sp., en guácima Guazuma ulmifolia Lam., 22.V.2013 (col. S.N. Myartseva, E. Ruiz-Cancino y J.M. Coronado-Blanco).
Hospederos. Aleyrodidae - Aleurothrixus floccosus (Maskell).

Distribución. Bolivia, Brasil, Colombia, El Salvador, Honduras, Puerto Rico, EU (California); México - Baja California Sur, Chiapas, Colima, Guerrero, Morelos, Oaxaca, San Luis Potosí, Sinaloa, Tamaulipas, Veracruz. Encarsia dimai Myartseva

Material. México, Tamaulipas, Altamira, 6 hembras, ex Aleurothrixus chivelensis (Sampson \& Drews) de Struthanthus sp., en guácima Guazuma ulmifolia Lam., 22.V.2013 (col. S.N. Myartseva).

Hospedero. Aleurothrixus chivelensis (Sampson \& Drews).

Distribución. México (Tamaulipas).

Encarsia altamira Myartseva, sp. nov.

(Figs 1-4)

Material. Holotipo hembra: México, Tamaulipas, Altamira, ex Aleurothrixus chivelensis (Sampson \& Drews) de Struthanthus sp., en guácima Guazuma ulmifolia Lam., 22.V.2013 (col. S.N. Myartseva, E. Ruiz-Cancino y J.M. Coronado-Blanco). Paratipos: mismos datos que el holotipo, seis hembras y cinco machos. El holotipo, dos pa-

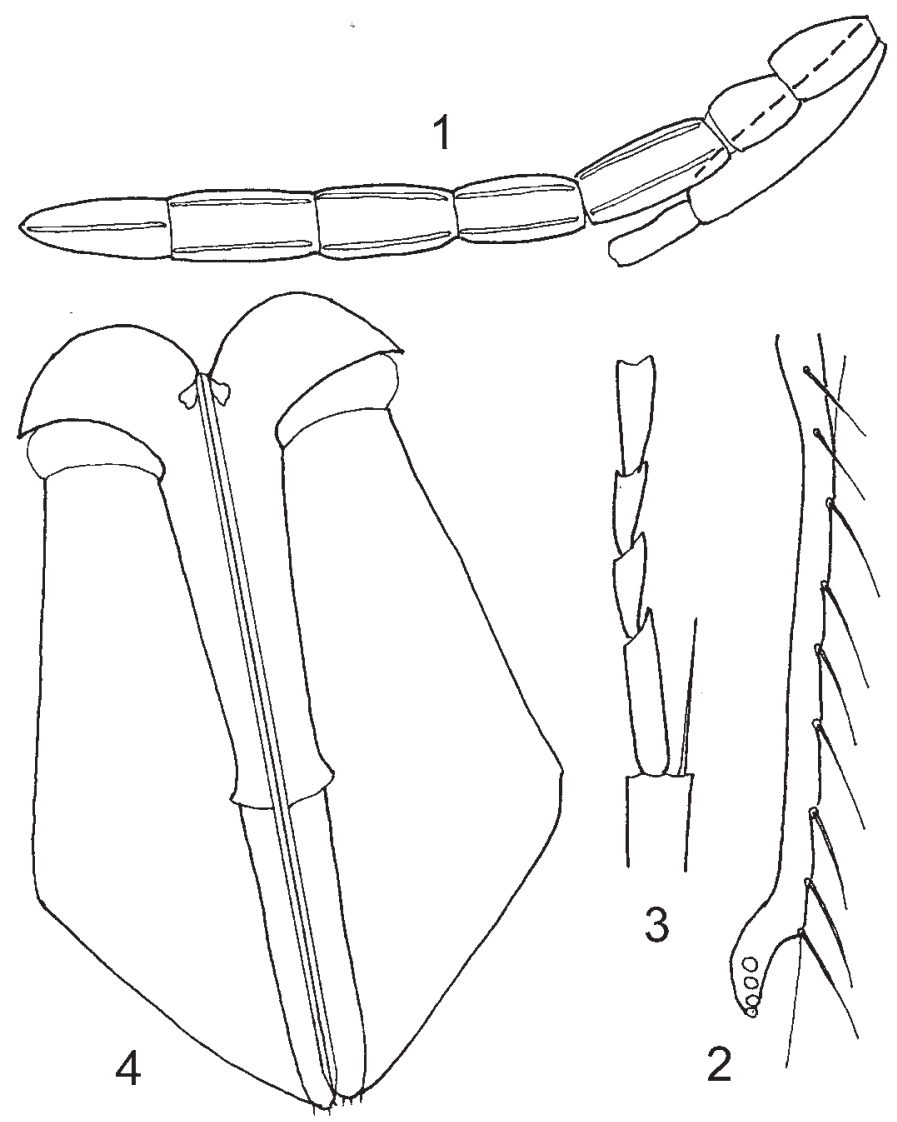

Figuras 1-4. Encarsia altamira, sp. nov., hembra: 1 - antena, 2 - vena marginal y vena estigmal del ala anterior, 3 - basitarso de la pata media, 4 - ovipositor. 
ratipos hembra y dos paratipos macho están depositados en UCRC, dos paratipos hembra y dos paratipos macho en UAT, y dos paratipos hembra y un paratipo macho en ZISP.

Etimología. El nombre de la nueva especie se deriva del Municipio de Altamira, Estado de Tamaulipas, donde se colectó este material.

Hembra. Longitud del cuerpo: 0.5-0.6 mm.

Coloración. Cabeza y cuerpo amarillo claro. Sulco malar y banda a través del occipucio en el nivel del foramen ligeramente ahumados. Ocelos rojizos. Cuerpo con las siguientes partes ahumadas: parte media del pronoto, lóbulo medio del mesoescudo a lo largo del margen anterior, axilas, propodeo, pecíolo, base del gáster, segmentos metasomales 5 y 6 completos, parte media del segmento 4; placas exteriores del ovipositor y ápice de los estiletes pardo oscuro. Alas anteriores hialinas y vena submarginal ligeramente ahumada. Patas blancuzcas.

Estructura. Cabeza no más ancha que el tórax, cerca de 1.5 veces tan ancha como alta. Frontovértice con estrías transversales débiles, 0.5 veces tan ancho como la anchura de la cabeza. Distancia entre los ocelos posteriores 0.6 veces tan larga como la distancia del ocelo al margen ocular. Ojos cerca de 1.4 veces tan largos como las mejillas. Mandíbula tridentada. Antenas insertadas inmediatamente al nivel del margen inferior de los ojos (Fig. 1). Distancia entre tórulos cerca de 0.8 veces tan larga como la distancia del tórulo al ojo. Radícula antenal 2.4 veces tan larga como ancha. Escapo 5.0 veces tan largo como ancho. Pedicelo cerca de 1.5 veces tan largo como ancho. Primer segmento funicular más corto que el pedicelo, cerca de 1.4 veces tan largo como ancho y cerca de 0.6 veces tan largo como el segundo segmento. Segundo y tercer segmentos 2.0 veces tan largos como anchos. Maza antenal de 3 segmentos, ligeramente más ancha y más corta que el funículo y el pedicelo juntos. Segmentos flagelares 2 al 6 con dos sensilas largas cada uno. Lóbulo medio del mesoescudo con 10-12 setas situadas simétricamente. Lóbulo lateral con 3 setas, axila con una seta. Escutelo cerca de 0.8 veces tan largo como el lóbulo medio y 1.9 veces tan ancho como largo. Sensilas escutelares placoideas ampliamente espaciadas. Distancia entre las setas escutelares anteriores 0.7 veces tan larga como la distancia entre las setas posteriores. Escultura del lóbulo medio del mesoescudo delgada y ampliamente reticulada. Alas anteriores uniformemente setosas y 2.4 veces tan largas como anchas, su fleco marginal cerca de 0.3 veces tan largo como la anchura alar máxima. Base alar con 4 setas. Vena marginal más larga que la submarginal (5:4), con 6-7 setas largas a lo largo del margen anterior (Fig. 2). Alas posteriores 7.6 veces tan largas como anchas, su fleco marginal 1.5 veces tan largo como la anchura alar máxima. Fórmula tarsal 5-45. Espuela de la tibia media tan larga como el basitarso (Fig. 3). Terguitos gástricos del 2 al 7 con 2, 2, 2, 4, 4 y 4 setas, respectivamente. Ovipositor no expuesto, cerca de 1.3 veces tan largo como la tibia media (Fig. 4). Tercera válvula cerca de 0.7 veces tan larga como el segundo valvífero.

Macho. Longitud del cuerpo: 0.5-0.6 mm.

Coloración. Cabeza y mesosoma como en la hembra. Metasoma negro parduzco, terguito 7 amarillento ahumado.

Estructura. Todos los segmentos flagelares antenales de la misma anchura, ligeramente más de dos veces tan largos como anchos y con dos sensilas largas cada uno; segmentos 5 y 6 separados.

Comentarios. Encarsia altamira sp. nov., pertenece al complejo de especies meritoria. Algunas especies de este complejo cercanamente relacionadas son difíciles de identificar y de distinguir morfológicamente, por lo que Polaszek et al. (2004) usaron análisis moleculares para cinco especies de este complejo. En el presente artículo se elaboró una clave que incluye las diferencias morfológicas de E. altamira sp. nov., con dichas especies.

\section{Clave para hembras del complejo de especies Encarsia meritoria}

1. Espuela de la tibia media 0.5 veces tan larga como el basitarso. Pedicelo ligeramente más largo que el segmento funicular 1 .......... californica Polaszek

- Espuela de la tibia media más de 0.5 veces tan larga como el basitarso. ................... 2

2. Segmento flagelar 6 elongado, 1.2 veces tan largo como el segmento 5. Pedicelo elongado, 1.2-1.3 veces tan largo como el primer segmento funicu-

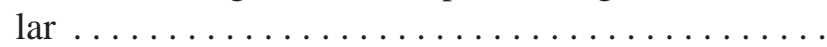
hispida De Santis

- Segmento flagelar 6 no elongado, tan largo como el segmento $5 \ldots \ldots \ldots \ldots \ldots \ldots \ldots \ldots$

3. Segundo segmento funicular más corto que el tercero. Tercera válvula 0.5 veces tan larga como el segundo valvífero. Escapo 1.6 veces tan largo como el sexto segmento funicular. Espuela de la tibia media 0.8 veces tan larga como el basitarso . . . . meritoria Gahan

- Segundo segmento funicular igual o más largo que el

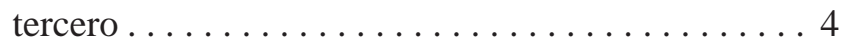

4. Placas exteriores del ovipositor pardas. Tercera válvula 0.7 veces tan larga como el segundo valvífero. Escapo 2.4 veces tan largo como el sexto segmento funicular. Espuela de la tibia media tan larga como el basitarso ................... altamira sp. nov. - Placas exteriores del ovipositor amarillas....... . 5 
5. Antenas pardas. Escutelo pardo claro. Tercera válvula $0.55-0.65$ veces tan larga como el segundo valvífero ................... dispersa Polaszek

- Antenas amarillo claro. Escutelo amarillo claro. Tercera válvula $0.75-0.80$ veces tan larga como el segundo valvífero................haitiensis Dozier

Agradecimientos. A los doctores Jacinto Treviño-Carreón y Arturo Mora-Olivo (Universidad Autónoma de Tamaulipas) por la identificación de las plantas, a Vicente E. Carapia-Ruiz (Universidad Autónoma del Estado de Morelos) e Ilya A. Gavrilov-Zimin (Zoological Institute, Russian Academy of Sciences, San Petersburgo, Rusia) por la identificación de las mosquitas blancas. Al Ing. Samuel Mireles Cepeda así como a Mariana y Ricardo Ruiz-Coronado por su colaboración en las colectas de mosquitas blancas. A la Universidad Autónoma de Tamaulipas por su apoyo.

\section{LITERATURA CITADA}

Arredondo Bernal, H. C. \& Rodríguez del Bosque, L. A. (Eds.). 2008. Casos de Control Biológico en México. Mundi Prensa México. 423 pp.

Greathead, D. 1986. Parasitoids in classical biological control, pp. 289-318. In: Waage, J. \& D. Greathead (eds.). Insect parasitoids. Academic Press, London. 389 pp.

Hayat, M. 1998. Aphelinidae of India (Hymenoptera: Chalcidoidea): a taxonomic revision. Memoirs on Entomology, International. Associated Publishers, Gainesville, Florida, U.S.A., 13: 1-416.

Hayat, M. 2012. Additions to the Indian Aphelinidae (Hymenoptera: Chalcidoidea) - III: The genus Encarsia Förster. Oriental Insects, 45: 202-274.

Heraty J, Woolley, J. \& Polaszek, A. 2007. Catalog of the Encarsia of the World. http://www.faculty.ucr.edu/ heraty/Encarsia.cat. pdf. $87 \mathrm{pp}$.

Huang, J. \& Polaszek, A. 1998. A revision of the Chinese species of Encarsia Förster (Hymenoptera: Aphelinidae): parasitoids of whiteflies, scale insects and aphids (Hemiptera: Aphelinidae, Diaspididae, Aphidoidea). Journal of Natural History, 32: 1825-1966.

Kim, J.W. \& Heraty, J. 2012. A phylogenetic analysis of the genera of Aphelininae (Hymenoptera: Aphelinidae), with a generic key and descriptions of new taxa. Systematic Entomology, 37: 497549.

Martin, J. H. 2005. Whiteflies of Belize (Hemiptera: Aleyrodidae).
Part 2 - a review of the subfamily Aleyrodinae Westwood. Zootaxa, 1098: 1-116.

Myartseva, S. N. 2007. Species of genus Encarsia Förster (Hymenoptera: Aphelinidae) - parasitoids of whiteflies (Hemiptera: Aleyrodidae) associated with Psidium guajava L. in Mexico, with key and description of new species. Biosystematica, 1: 7-19.

Myartseva, S. N. 2014. A new species of Encarsia Förster (Hymenoptera: Chalcidoidea: Aphelinidae) from Altamira, Tamaulipas, Mexico. Proceedings of the Russian Entomological Society. St Petersburg, 85: 165-169.

Myartseva, S. N. \& Evans, G. A. 2008. Genus Encarsia Förster of Mexico (Hymenoptera: Chalcidoidea: Aphelinidae). A revision, key and description of new species. Serie Avispas Parasíticas de Plagas y Otros Insectos, 3. Universidad Autónoma de Tamaulipas, Ciudad Victoria, México. 320 p.

Myartseva, S. N., Ruiz-Cancino, E. \& Coronado-Blanco, J. M. 2012. Aphelinidae (Hymenoptera: Chalcidoidea) de importancia agrícola en México. Revisión y claves. Serie Avispas Parasíticas de Plagas y otros Insectos No. 8. Universidad Autónoma de Tamaulipas. México. 413 p. Publicación en CD.

Myartseva, S.N., Ruiz-Cancino, E. \& Coronado-Blanco, J. M. 2013. Four new species of Aphelinidae (Hymenoptera: Chalcidoidea) from Mexico. Zootaxa, 3641: 223-232.

Noyes, J. S. 2013. Universal Chalcidoidea Database [online]. Worldwide Web electronic publication. www.nhm.ac.uk/entomology/ chalcidoids/index.html (last updated: October 2013).

Polaszek, A., Manzari, S. \& Quicke, D. L. 2004. Morphological and molecular taxonomic analysis of the Encarsia meritoria speciescomplex (Hymenoptera, Aphelinidae), parasitoids of whiteflies (Hemiptera, Aleyrodidae) of economic importance. Zoologica Scripta 33: 403-421.

Polaszek, A., Hernández-Suárez, E.M., Manzari, S., Pedata, P.A. \& Schmidt, S. 2009. Megadiversity of Encarsia (Chalcidoidea, Aphelinidae): macroevolution in a microhymenopteran. Memoria de Taller Internacional sobre Recursos Naturales, 21 al 23 de Octubre, 2009, Cd. Victoria, Tamaulipas, México: 87-92.

Rosen, D. \& DeBach, P. 1990. Ectoparasites, pp. 99-120. In: Rosen, D. (Ed.). Armored scale insects. Vol. 4B. Elsevier, Amsterdam, The Netherlands.

Sampson, W. W. \& Drews, E. A. 1941. Fauna Mexicana IV. A review of the Aleyrodidae of Mexico. Anales de la Escuela Nacional de Ciencias Biológicas, 2: 143-189.

Schmidt, S. \& Polaszek, A. 2007. Encarsia or Encarsiella? - redefining generic limits based on morphological and molecular evidence (Hymenoptera, Aphelinidae). Systematic Entomology, 32: 81-94. 